\title{
Recanalization of an obstructive pancreaticojejunal anastomosis with direct visualization by using antegrade peroral pancreatoscopy
}

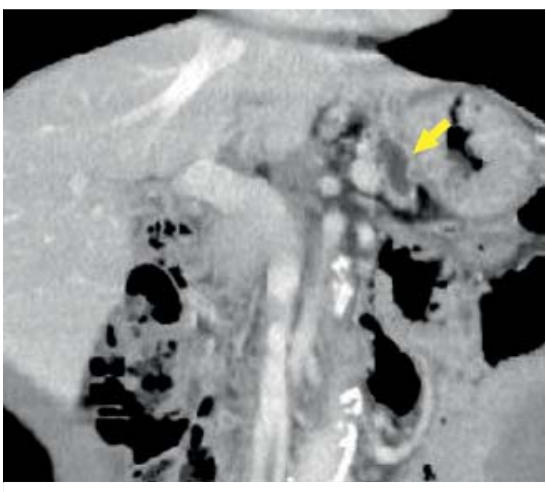

- Fig. 1 Contrast-enhanced computed tomography (coronal views) showing the dilated main pancreatic duct of the remnant pancreas (arrow).

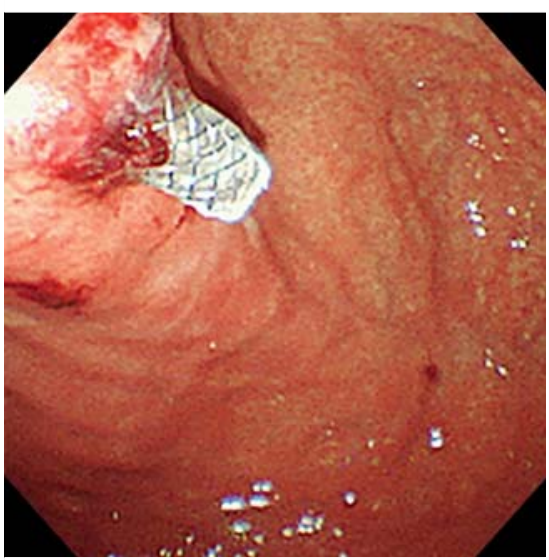

- Fig.3 A 6-mm fully covered self-expandable metallic stent was inserted across the pancreaticogastrostomy.

A 60-year-old man came to our hospital complaining of upper abdominal pain possibly due to stenosis of a pancreaticojejunal anastomosis with upstream dilation of the main pancreatic duct ( Fig.1). Because an endoscopic transluminal approach via the afferent loop failed, we performed endoscopic ultrasound (EUS)-guided pancreatic drainage with a 19-gauge needle (EZ Shot 3 Plus; Olympus Co., Tokyo, Japan). However, no contrast medium flowed out of the dilated main pancreatic duct to the jeju-
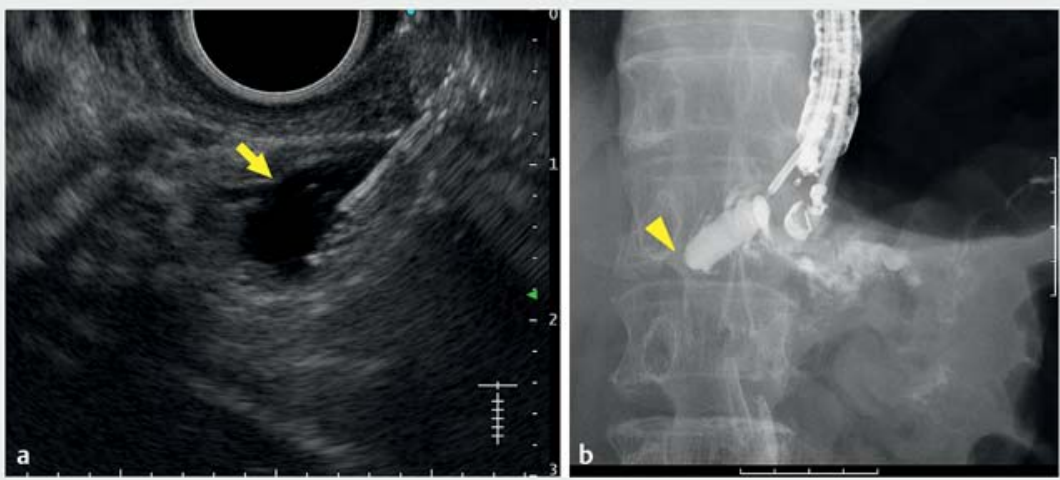

- Fig. 2 Endoscopic ultrasound-guided pancreatic drainage. a The dilated main pancreatic duct (arrow) was punctured with a 19-gauge needle under endoscopic ultrasound guidance. b From fluoroscopy, no contrast medium flowed out of the dilated pancreatic duct (arrowhead).
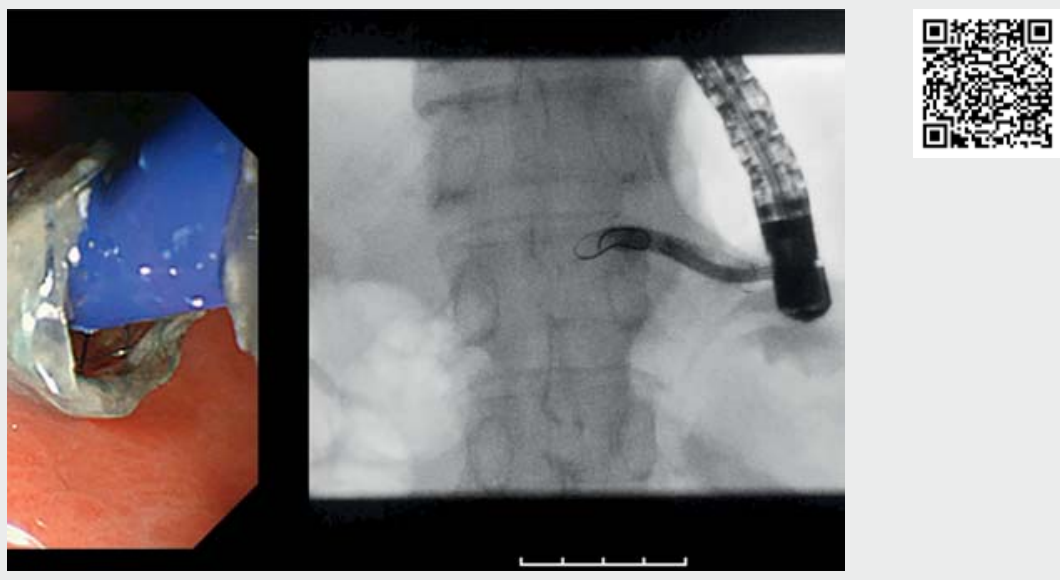

Video 1 Recanalization of the stenosis of a pancreaticojejunal anastomosis under direct visualization by using antegrade peroral pancreatoscopy via endoscopic ultrasound-guided pancreaticogastrostomy.

num ( Fig. 2), and a 0.025-inch guidewire could not be inserted across the anastomosis. A 7-Fr plastic stent was exchanged, 1 month later, for a 6-mm fully covered self-expandable metallic stent (Niti-S Biliary S-type Stent, Century Medical Co., Ltd., Tokyo, Japan) across the pancreaticogastrostomy to perform per- oral pancreatoscopy (POPS) (\ Fig. 3). A SpyGlass DS system (Boston Scientific Co., Marlborough, Massachusetts, USA) was used to perform POPS to visualize the anastomosis from the inside of the main pancreatic duct ( Video 1 ). We found the duct completely obstructed at the anastomotic site and covered with 

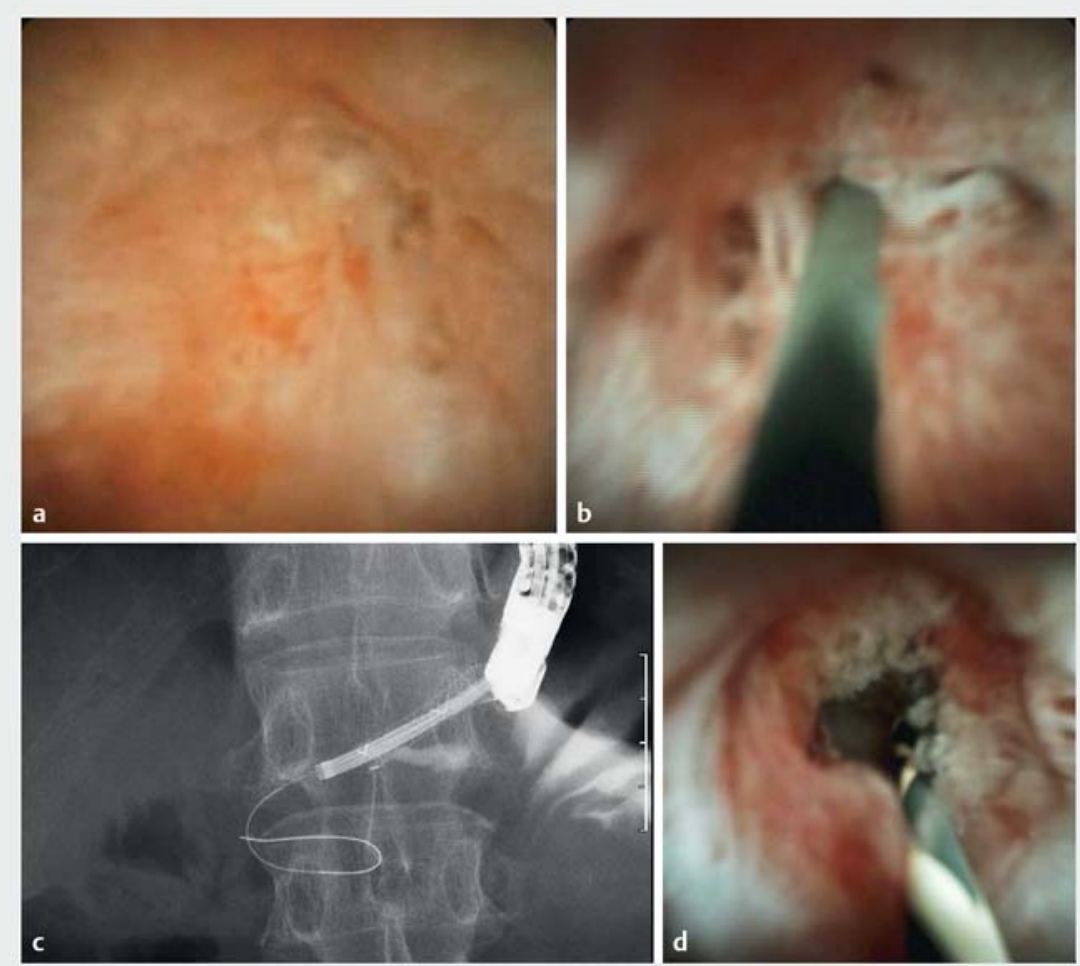

- Fig. 4 Peroral pancreatoscopy (POPS) using a SpyGlass DS system. a POPS revealed complete obstruction of the main pancreatic duct at the anastomotic site, which was covered with fibrotic tissues. $\mathbf{b}$ The fibrotic tissues were partially broken by poking repeatedly with a guidewire. c A guidewire could be passed through the anastomosis. $\mathbf{d}$ After the anastomosis was dilated by use of a dilator and balloon catheter along the guidewire, POPS images confirmed that the anastomosis site was adequately dilated.

fibrotic tissues ( $\triangleright$ Fig. 4 ). It was difficult to break through this obstruction even with POPS guidance. However, repeated poking with a guidewire partially broke the fibrotic tissues and a guidewire could finally be passed through the anastomosis. After dilation of this anastomosis using a 7-Fr catheter and a $6-\mathrm{mm}$ balloon catheter, contrast medium immediately flowed from the main pancreatic duct to the jejunum. No procedure-related adverse events were observed, and the abdominal symptoms improved after treatment.

Although the efficacy of EUS-guided pancreatic drainage for stenosis of the pancreaticojejunal anastomosis has been described [1,2], the procedure is still challenging. Recently, the usefulness of cholangioscopy for stenosis of the bilioenteric anastomosis has been reported $[3,4]$. Therefore, direct visualization using POPS via EUS-guided pancreaticoventions have failed.

Endoscopy_UCTN_Code_TTT_1AR_2AG

\section{Competing interests} interest.

\section{The authors}

Yujiro Kawakami ${ }^{1,2}$, Shinsuke Koshita ${ }^{1}$, Yoshihide Kanno', Takahisa Ogawa' ${ }^{1}$, Toji Murabayashi ${ }^{1}$, Hiroshi Nakase ${ }^{2}$, Kei Ito ${ }^{1}$

1 Department of Gastroenterology, Sendai City Medical Center, Sendai, Japan

2 Department of Gastroenterology and Hepatology, Sapporo Medical University School of Medicine, Sapporo, Japan
Corresponding author

\section{Yujiro Kawakami, MD}

Department of Gastroenterology, Sendai City Medical Center, 5-22-1, Tsurugaya, Miyagino-ku, Sendai 9830824, Japan

Fax: +81-22-252-9431

yujiro.kawakami@gmail.com

References

[1] Matsunami Y, Itoi T, Sofuni A et al. Evaluation of a new stent for EUS-guided pancreatic duct drainage: long-term follow-up outcome. Endosc Int Open 2018; 6: E505E512

[2] Ogura T, Nishioka N, Yamada M et al. Twostep endoscopic ultrasound-guided rendezvous technique combined with antegrade electrohydraulic lithotripsy for a huge pancreatic duct stone. Endoscopy 2019; 51: E149-E150

[3] Fujii Y, Koshita S, Ito K. Percutaneous transhepatic cholangioscopy using SpyGlassDS for an anastomotic stenosis after choledochojejunostomy. Dig Endosc 2018; 30: 806807

[4] Hakuta R, Kogure H, Nakai Y et al. Successful guidewire placement across hilar biliary stricture after decreased donor liver transplantation using new digital cholangioscopy. Endoscopy 2018; 50: E54-E56

Bibliography

gastrostomy appears to be a promising alternative method if fluoroscopic inter-

DOI https://doi.org/10.1055/a-1133-4304

Published online: 27.3.2020

Endoscopy 2020; 52: E376-E377

(c) Georg Thieme Verlag KG

Stuttgart · New York

ISSN 0013-726X

The authors declare they have no conflict of

\section{ENDOSCOPY E-VIDEOS}

https://eref.thieme.de/e-videos

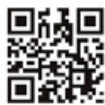

Endoscopy E-Videos is a free access online section, reporting on interesting cases and new techniques in gastroenterological endoscopy. All papers include a high quality video and all contributions are freely accessible online.

This section has its own submission website at https://mc.manuscriptcentral.com/e-videos 